ARTIGO ORIGINAL

\title{
Correlação entre dimensões das árvores de eucalipto em alto fuste e talhadia
}

\author{
Correlation between dimensions of eucalyptus trees in high fuses and wood
}

Geraldo Magela Pereira Filho ${ }^{1}$ (D), Laércio Antônio Gonçalves Jacovine ${ }^{1}$ (), Bruno Leão Said Schettini ${ }^{1}$ (ㄷ), Haroldo Nogueira de Paiva ${ }^{1}$ (D), Fernando Palha Leite ${ }^{1}$, Paulo Henrique Villanova ${ }^{1}$ (D), Samuel José Silva Soares da Rocha ${ }^{1}$ (1) , Hélio Garcia Leite ${ }^{1}$ (1)

'Universidade Federal de Viçosa - UFV, Viçosa, MG, Brasil

Como citar: Pereira Filho, G. M., Jacovine, L. A. G., Schettini, B. L. S., Paiva, H. N., Leite, F. P., Villanova, P. H., Rocha, S. J. S. S., \& Leite, H. G. (2020). Correlação entre dimensões das árvores de eucalipto em alto fuste e talhadia. Scientia Forestalis, 48(125), e3212. https://doi.org/10.18671/scifor.v48n125.24

\begin{abstract}
Resumo
O regime de talhadia tem sido cada vez mais utilizado por acarretar em ganhos econômicos e ambientais quando comparado ao alto fuste. No entanto, o conhecimento da influência das características do alto fuste no crescimento e produtividade de florestas sob o regime de talhadia ainda precisa ser ampliado. O objetivo do estudo foi avaliar a correlação entre as dimensões das árvores em regime de alto fuste e talhadia assim como avaliar a distribuição diamétrica e a uniformidade em dois povoamentos florestais localizados nas regiões de Belo Oriente e Virginópolis, em MG. O regime de alto fuste foi implantado no ano de 2015 e o clone utilizado foi o Eucalyptus urophylla $\times$ Eucalyptus grandis, com espaçamentos de $3 \times 2 \mathrm{~m}$ e de $3 \times 3 \mathrm{~m}$, respectivamente. O regime de talhadia foi implantado no sétimo ano, após a colheita das árvores. A coleta das variáveis dendrométricas (DAP e altura) foi realizada aos 40 e 80 meses para o alto fuste e aos 40 meses (após a desbrota) para a talhadia. $O$ volume foi estimado utilizando equações alométricas ajustadas para cada povoamento. Não houve correlação pelo método de Pearson ( $p$-valor $>0,05$ ) entre as variáveis avaliadas (DAP, altura e volume) nos regimes de alto fuste e talhadia. As árvores, em sua maioria, permaneceram nas mesmas classes diamétricas (amplitude de $5 \mathrm{~cm}$ ) de um regime para outro, na mesma idade de avaliação. No entanto, a uniformidade foi diferente entre os regimes. Assim, pode-se concluir que as árvores dominantes no alto fuste tendem a ser dominadas no regime de talhadia.
\end{abstract}

Palavras-chave: Manejo florestal; Variáveis dendrométricas; Uniformidade de plantio.

\begin{abstract}
The coppice method has been increasingly used for economic and environmental gains when compared to the high stem. However, the knowledge of the influence of the characteristics of the high stem on the growth and productivity of forests under the coppice regime still needs to be expanded. The objective of the study was to evaluate the correlation between the dimensions of the trees with high stem and coppice regimes as well as to evaluate the diametrical distribution and the uniformity in two forest stands located in the regions of Belo Oriente and Virginópolis, MG. The high stem regime was implanted in the year 2015 and the clone used was Eucalyptus urophylla $x$ Eucalyptus grandis, spaced $3 \times 2 \mathrm{~m}$ and $3 \times 3 \mathrm{~m}$, respectively. The woodcut regime was implemented in the seventh year after harvesting the trees. The collection of the dendrometric variables (dbh and height) was performed at 40 and 80 months for the high stem and at 40 months (after the cutting) for the coppice. The volume was estimated using allometric equations adjusted for each settlement. There was no correlation by the Pearson method ( $p$-value>0.05) between the variables evaluated ( $\mathrm{dbh}$, height and volume) in the high-stem and coppice regimes. Most trees remained in the same diametric classes (range of $5 \mathrm{~cm}$ ) from one regime to another at the same evaluation age. However, there was a difference between the uniformity of the regimens. Thus, it can be concluded that the dominant trees in the upper stem can be dominated in the coppice regime.
\end{abstract}

Fonte de financiamento: Nenhuma.

Conflito de interesse: Nada a declarar.

Autor correspondente: blsschettini@gmail.com

Recebido: 11 dezembro 2018.

Aceito: 17 junho 2019.

Editor: Francides Gomes da Silva Júnior.

(c) (†) Este é um artigo publicado em acesso aberto (Open Access) sob a licença Creative Commons Attribution, que permite uso, distribuição e reprodução c) em qualquer meio, sem restrições desde que o trabalho original seja corretamente citado. 
Keywords: Forest management; Dendrometric variables; Planting uniformity.

\section{INTRODUÇÃO}

No Brasil, a condução de plantios comerciais de eucalipto por meio da talhadia é cada vez mais comum (Azevedo et al., 2011), evitando custos com mudas, preparo de solo e plantio (Indústria Brasileira de Árvores, 2017). A regeneração de povoamentos florestais por meio da brotação em comparação com a regeneração por meio do alto fuste pode ser $27,12 \%$ mais barata até a realização da colheita (Rode et al., 2015). O gênero Eucalyptus, por apresentar muitas espécies com capacidade de regenerar por meio da brotação de cepas, possibilita a adoção do manejo por talhadia acarretando em benefícios econômicos e ambientais uma vez que não são necessárias intervenções físicas no solo (Gadelha et al., 2015).

A taxa de crescimento inicial de brotações é superior à de povoamentos em alto fuste, podendo resultar em antecipação da idade em que ocorre a produtividade máxima (Cacau et al., 2008). A produção obtida em povoamentos de eucalipto manejados por talhadia é, em princípio, semelhante à do povoamento original, desde que a disponibilidade de fatores de crescimento (água, luz, nutrientes e temperatura) não seja diminuída (Gonçalves et al., 2014). Entretanto, no Brasil, o volume de madeira produzido no alto fuste é $30 \%$ superior ao da talhadia (Guedes et al., 2011), uma vez que a brotação tem idade referente ao plantio inicial e não ao aparecimento do broto, o que pode resultar na queda de vigor da planta (Faria et al., 2002). Essa queda de produtividade varia em função do genótipo (clone), sobrevivência, altura das brotações, época de corte, manejo de formigas-cortadeiras e cupins, época de corte do plantio anterior, competição com ervas daninhas, época e intensidade de desbrota e dos danos causados à cepa e ao solo durante a colheita (Stape et al., 2010).

Para atingir a produtividade potencial de um povoamento é necessário conhecer a qualidade do site, genética das árvores (Resende et al., 2018), garantir a qualidade das atividades florestais e a exatidão das recomendações técnicas (Stape et al., 2010). Qualquer falha na recomendação técnica ou na qualidade da execução das atividades pode proporcionar distribuição heterogênea de recursos pela área manejada, o que pode resultar em povoamentos heterogêneos quanto ao crescimento das árvores.

A variação no crescimento das árvores dentro do povoamento faz com que as árvores maiores obtenham mais recursos e cresçam mais rápido que as árvores menores, levando ao aumento na variação do tamanho das árvores (Otto et al., 2014). Portanto, a garantia da manutenção da uniformidade do povoamento aumenta a probabilidade de obtenção da produtividade atingível do sítio (Stape et al., 2010).

Com o aumento da importância da talhadia, faz-se necessário ampliar o conhecimento sobre os fatores que afetam o crescimento da brotação e seu impacto sobre a possível redução do potencial de produtividade. Assim, o objetivo deste trabalho foi: i) avaliar a correlação entre as dimensões das árvores no final da rotação em regime de alto fuste e na condução da talhadia; e ii) avaliar a distribuição de diâmetros e a uniformidade do povoamento no regime de alto fuste e de talhadia.

\section{MATERIAL E MÉTODOS}

$\mathrm{O}$ estudo foi realizado em povoamentos florestais com clones de Eucalyptus urophylla $\mathrm{x}$ Eucalyptus grandis, em duas regiões, pertencentes à empresa Celulose Nipo-Brasileira S.A. (CENIBRA S.A.). A primeira no município de Belo Oriente, cujo clima é caracterizado como Aw (Köppen), temperado chuvoso-mesotérmico, com precipitação média anual de $1.208 \mathrm{~mm}$, temperatura média anual de $25,2^{\circ} \mathrm{C}$ e umidade relativa média de $65,2 \%$. A região situa-se na latitude $19^{\circ} 10^{\prime} \mathrm{S}$ e longitude de $42^{\circ} 20^{\prime} \mathrm{W}$, com altitude variando de 220 a $425 \mathrm{~m}$, em situação de encosta. A segunda região, localizada no munícipio de Virginópolis, situa-se na latitude de $18^{\circ} 45^{\prime} \mathrm{S}$ e longitude de $42^{\circ} 34^{\prime} \mathrm{W}$, com altitude variando de 742 a $992 \mathrm{~m}$, também em situação de encosta. O clima é classificado como Cwa (Köppen), com verão chuvoso e inverno seco, com temperatura média anual de $22,2^{\circ} \mathrm{C}$ e precipitação média anual de $1.065 \mathrm{~mm}$.

O plantio, em Belo Oriente, foi realizado em abril de 2005 no espaçamento de 3,00 × 2,00 m, em Latossolo Vermelho. Em Virginópolis, o plantio foi realizado em novembro 
de 2005, no espaçamento de 3,00 × 3,33 m, em Latossolo Amarelo. O preparo do solo, nas duas áreas, foi feito com uso de subsolador. A adubação do plantio foi realizada de acordo com a recomendação técnica da empresa, com a aplicação de $1.500 \mathrm{~kg} \mathrm{ha}^{-1}$ de calcário aplicado a lanço em área total, antes do plantio, $100 \mathrm{~g} \mathrm{ha}^{-1}$ de fertilizante NPK (06-30-06), aplicado imediatamente após o plantio, em duas covetas laterais. A adubação de cobertura, 4 meses após o plantio, foi realizada com aplicação de $300 \mathrm{~kg} \mathrm{ha}^{-1}$ de NPK (06-10-29).

A colheita das árvores ocorreu sete anos após o plantio, com o uso do Harvester e Forwarder. O corte das árvores foi realizado deixando as cepas com altura entre 5 e $15 \mathrm{~cm}$. Os resíduos da colheita permaneceram espalhados no local do corte. A desbrota foi feita com uma cavadeira, quando os brotos apresentavam altura média entre 1,0 e 2,0 m, deixando o broto mais vigoroso por cepa. A adubação na talhadia variou de acordo com as características de fertilidade do solo. Em Belo Oriente foram aplicados $400 \mathrm{~kg} \mathrm{ha}^{-1}$ do fertilizante NPK (06-10-29) e $700 \mathrm{~kg} \mathrm{ha}^{-1}$ de calcário. Em Virginópolis foram aplicados $600 \mathrm{~kg} \mathrm{ha}^{-1}$ do fertilizante NPK (06-10-29) e $1000 \mathrm{~kg} \mathrm{ha}^{-1} \mathrm{de}$ calcário. A aplicação do calcário foi realizada até 2 meses após a desbrota. O controle de broto ladrão foi realizado até 5 meses após a realização da desbrota.

Parcelas contendo 36 árvores foram instaladas em talhões comerciais da empresa, perfazendo 24 parcelas (área total de cada parcela: $216 \mathrm{~m}^{2}$ ) em Virginópolis e 27 (área total de cada parcela: 359,64 $\mathrm{m}^{2}$ ) parcelas em Belo Oriente. O diâmetro a 1,3 m de altura (DAP) e a altura $(\mathrm{Ht})$ das plantas na área útil da parcela foram medidos aos 40 e 80 meses após o plantio e aos 40 meses após a desbrota. Árvores com DAP superior a $5 \mathrm{~cm}$ foram medidas no alto fuste, sendo que na talhadia, independente do DAP, todos os indivíduos foram mensurados. A sequência de medição das árvores foi mantida de uma medição para outra, com o objetivo de correlacionar as características analisadas em uma mesma árvore no alto fuste e na talhadia.

O volume individual das árvores foi determinado com base na equação, ajustada pela empresa, para cada região e manejo. Para a região de Belo Oriente foram usadas as equações: $\ln (\mathrm{V})=-10,2924+1,7653 \times \ln (\mathrm{DAP})+1,2206 \times \ln (\mathrm{Ht})$, para o alto fuste, e $\ln (\mathrm{V})=-10,3272+1,8206 \times \ln (\mathrm{DAP})+1,1756 \times \ln (\mathrm{Ht})$, para a talhadia. Já para a região de Virginópolis foram utilizadas as equações: $\ln (\mathrm{V})=-10,1399+1,8683 \times \ln (\mathrm{DAP})+1,0777$ $\times \ln (\mathrm{Ht})$, para o alto fuste, e $\ln (V)=-10,2420+1,8097 \times \ln (\mathrm{DAP})+1,1627 \times \ln (\mathrm{Ht})$, para a talhadia.

Todas as variáveis avaliadas continham as informações da mesma árvore na primeira e segunda rotação. Para as variáveis DAP, altura e volume individual, avaliou-se a correlação do efeito da dimensão das árvores no alto fuste sob a dimensão das árvores na talhadia, por meio da correlação de Pearson, adotando-se o nível de significância de 5\%. Para o processamento dos dados foi utilizado o programa estatístico Statistica versão 12 (Statsoft Inc., 2014).

Adotou-se classes de diâmetro com amplitude de $5 \mathrm{~cm}$ para agrupamento das árvores das parcelas em classes diamétricas. Análises estatísticas descritivas foram feitas para verificar mudanças da estrutura diamétrica dos povoamentos e a dinâmica de crescimento das árvores. As distribuições de diâmetro foram utilizadas para inferir sobre a uniformidade do povoamento, para constatar se a uniformidade do povoamento no alto fuste tem relação com a uniformidade na talhadia.

\section{RESULTADOS E DISCUSSÕES}

Não houve correlação entre o crescimento das árvores no alto fuste e na talhadia para nenhuma das variáveis avaliadas ( $p$-valor $>0,05$ ). A variável que apresentou menor coeficiente de correlação foi volume individual, seguido pelo DAP e pela altura (Figura 1). 

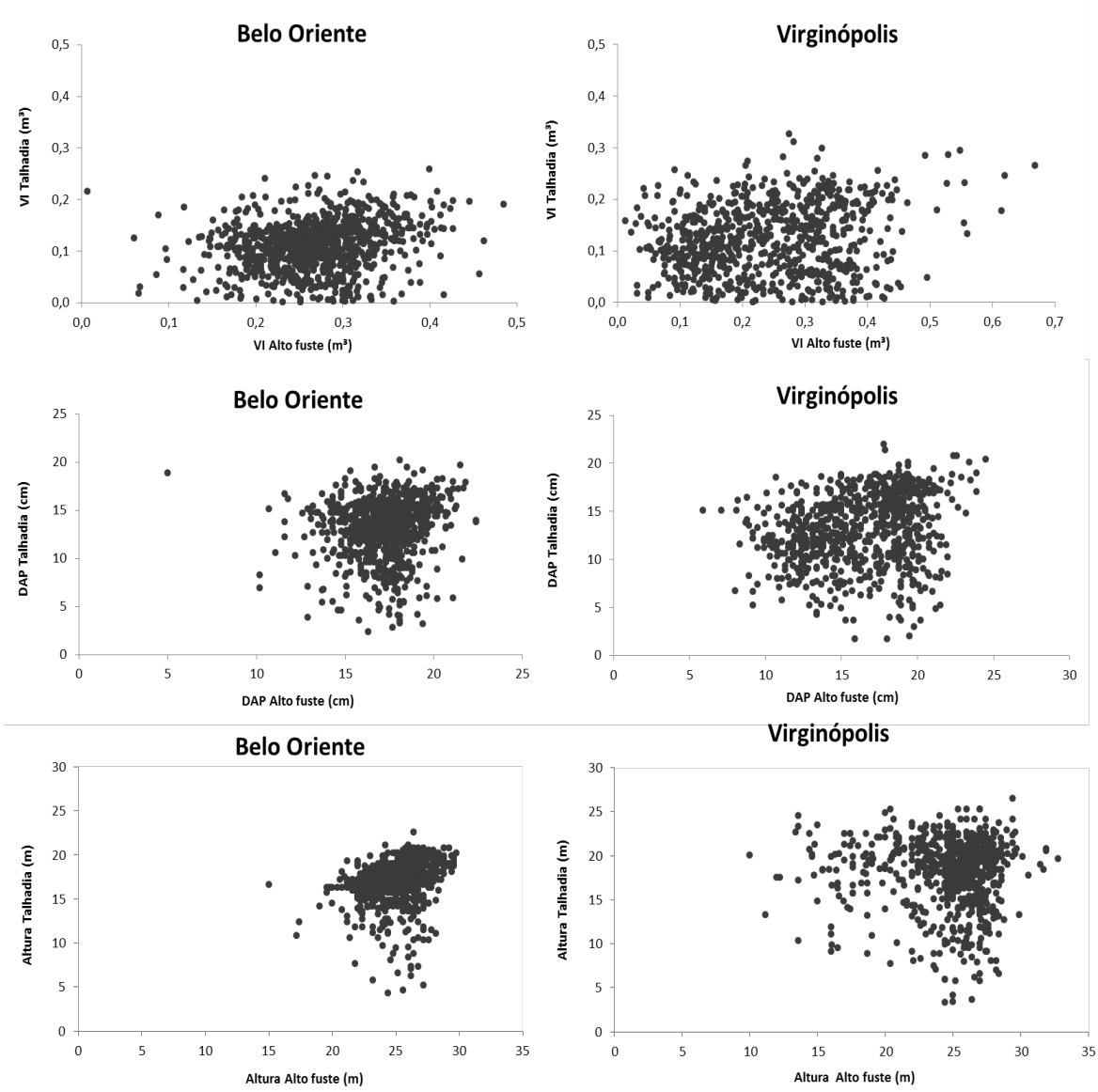

Figura 1: Relações entre árvores de E. urophylla $\times$ E. grandis para as variáveis volume individual, DAP e altura, aos 80 meses no alto fuste e aos 40 meses na talhadia.

Os valores de coeficiente de determinação $\left(R^{2}\right)$ para as variáveis Volume, DAP e altura foram calculados aos 40 e 80 meses de idade do alto fuste em relação a talhadia, não havendo correlação entre as variáveis avaliadas (Tabela 1).

Tabela 1: Coeficiente de determinação $\left(R^{2}\right)$ para as variáveis Volume, DAP e altura nas regiões de Belo Oriente e Virginópolis

\begin{tabular}{cccc}
\hline Região & Idade (Meses) & Variável & R2 (\%) \\
\hline \multirow{2}{*}{ Belo Oriente } & \multirow{2}{*}{80} & Volume & 4,73 \\
& & DAP & 1,89 \\
& & Altura & 7,83 \\
Virginópolis & \multirow{2}{*}{40} & Volume & 3,16 \\
& & DAP & 3,16 \\
& \multirow{2}{*}{40} & Altura & 0,04 \\
Belo Oriente & Volume & 8,18 \\
& & DAP & 12,11 \\
& \multirow{2}{*}{40} & Altura & 14,38 \\
Virginópolis & Volume & 3,27 \\
& & DAP & 7,42 \\
& & Altura & 6,42 \\
\hline
\end{tabular}

Diferentes são os fatores que podem contribuir para que as variáveis analisadas não apresentassem correlação entre o alto fuste e a talhadia. Árvores mais grossas apresentam maior acúmulo de reservas de carboidratos no sistema radicular, o que permite o aumento 
do vigor da brotação (Mroz et al., 1985; Teixeira et al., 2002), porém fatores extrínsecos às condições fisiológicas das brotações podem ter afetado o crescimento das árvores conduzidas no manejo por talhadia.

Os povoamentos apresentaram diferença na mortalidade das brotações, sendo esta de $7,5 \%$ para a região de Belo Oriente e 13,1\% para Virginópolis. Ao analisar a mortalidade na talhadia, a partir da taxa de árvores mortas por classe diamétrica, no alto fuste aos 80 meses, também foi observada diferença entre os plantios (Figura 2). Na região de Belo Oriente, a classe de 10 a $15 \mathrm{~cm}$ foi aquela com maior porcentual de cepas mortas, devendo ser ressaltado que quanto maior o diâmetro das árvores no alto fuste menor foi a taxa de mortalidade das cepas. Para o plantio na região de Virginópolis, a menor $(5$ a $10 \mathrm{~cm})$ e a maior classe diamétrica $(20$ a $25 \mathrm{~cm}$ ) foram as que apresentaram maior porcentual de cepas sem brotação.
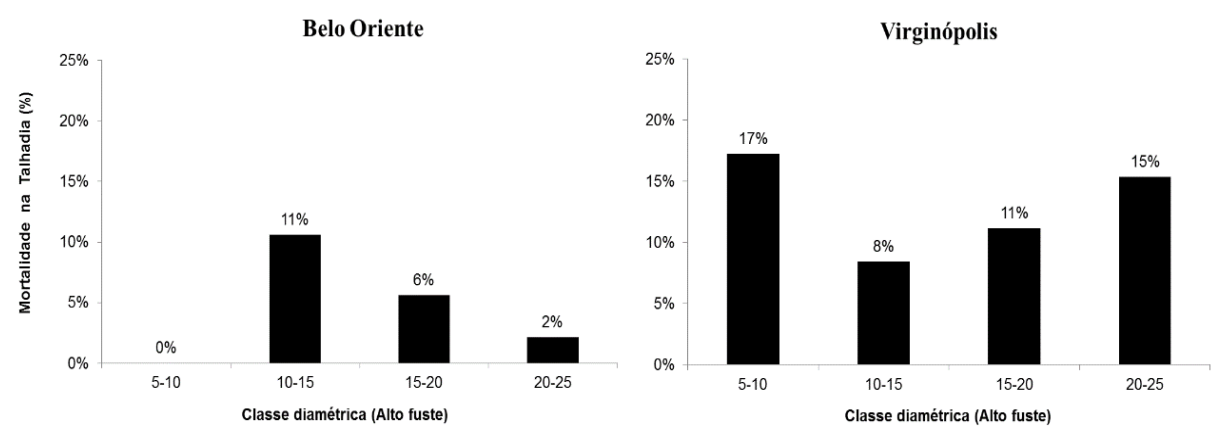

Figura 2: Mortalidade das cepas, pós-corte, por classe diamétrica do alto fuste aos 80 meses.

O maior crescimento inicial das brotações se deve à utilização de reservas orgânicas e inorgânicas nas cepas ou nas raízes. Posteriormente, quando passam a depender diretamente do solo, o sistema radicular desenvolvido favorece a absorção de água e nutrientes, aumentando a taxa de crescimento da parte aérea das brotações (Walters et al., 2005). Após a desbrota, para o desenvolvimento dos brotos remanescentes, a planta requer maior quantidade de água e nutrientes do solo (Cacau et al., 2008). Qualquer falha na qualidade da realização da atividade de adubação (Simões et al., 2012) ou na disponibilidade de nutrientes no solo (Christina et al., 2018) da área manejada compromete o crescimento das brotações, o que afeta sua produtividade final, podendo gerar plantas dominadas dentro do povoamento.

A realização de tratos silviculturais inadequados pode prejudicar a brotação e contribuir para o aumento da mortalidade e a redução do crescimento dos brotos selecionados (Retslaff et al., 2015). Assim, a realização de operações florestais de acordo com a recomendação técnica para a área e com o padrão de qualidade desejado é fundamental para o bom desenvolvimento dos brotos. Aparício et al. (2010), estudando a influência da matocompetição em clones de E. urophylla $\times$ E. grandis, verificaram que o DAP foi a variável mais sensível.

A época do ano em que é realizado o corte das árvores em alto fuste influencia o resultado final da regeneração por meio da brotação de cepas, uma vez que temperaturas extremas, ausência de chuvas e insolação excessiva reduzem o número de brotos e a sua qualidade (Ferrari et al., 2004). Quando a colheita ocorre no período de seca, a morte das raízes finas pode ser mais intensa, o que pode reduzir o vigor das cepas (Reis \& Reis, 1997) e, portanto, prejudicar o crescimento da brotação.

A classe de diâmetro com maior frequência de indivíduos nos dois regimes, aos 40 meses, foi a mesma (10-15 cm) (Figura 3). 


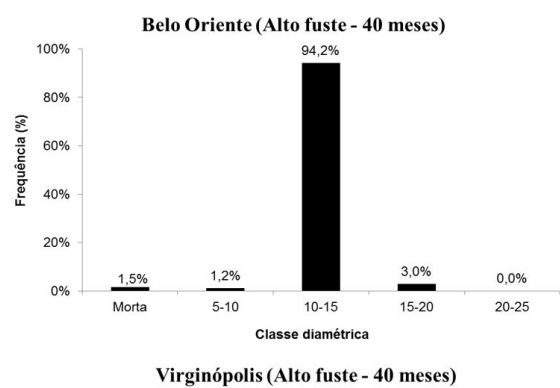

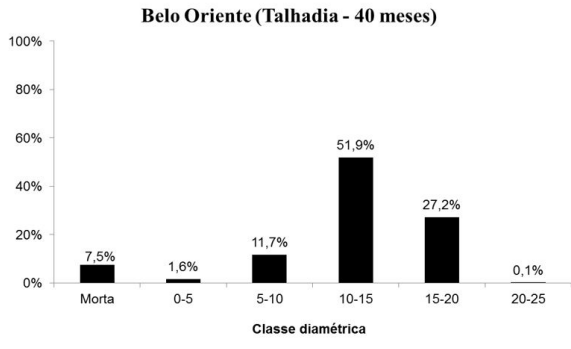

Virginópolis (Talhadia - 40 meses)

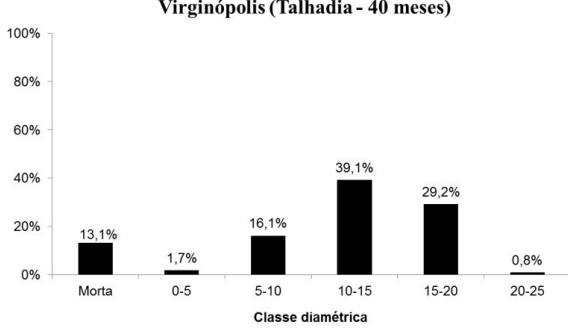

Figura 3: Distribuição diamétrica das árvores, aos 40 meses de idade, nos regimes em alto fuste e talhadia, para as regiões estudadas.

As operações que envolvem a colheita de madeira, podem acarretar significativas perdas para áreas que se pretende conduzir talhadia (Ferrari et al., 2004). A compactação causada pelo tráfego de máquinas durante a colheita reduz em até dois terços o volume total de madeira, da área de rebrota, nas linhas com maiores compactações de solo argiloso (Dedecek \& Gava, 2005). A movimentação das máquinas dentro dos talhões pode afetar a sobrevivência da brotação e o crescimento inicial dos brotos. O tráfego das máquinas pode resultar em danos mecânicos às cepas (Andrade et al., 2000), como esmagamento, rachadura e perda de casca, reduzindo, assim, seu potencial de brotação. Outro fator que pode influenciar a brotação é a cobertura das cepas pelos resíduos resultantes da colheita, como folhas, galhos e casca proveniente do descascamento da madeira no campo, que bem manejados aumentam a produtividade dos plantios (Rocha et al., 2016).

A maioria das árvores permaneceu nas mesmas classes diamétricas de um regime de manejo para outro, na mesma idade. Árvores pertencentes a menores classes de diâmetro no alto fuste foram as que apresentaram maior migração porcentual de indivíduos para classes diamétricas imediatamente superiores na talhadia. Árvores dominadas na talhadia, em sua maioria, foram formadas por árvores de classes diamétricas superiores no regime de alto fuste (Tabela 2).

Tabela 2: Distribuição porcentual dos diâmetros, considerando a dinâmica da mudança das árvores em classes diamétricas do manejo de alto fuste para a talhadia, para a região de Belo Oriente e Virginópolis, aos 40 meses de idade

\begin{tabular}{|c|c|c|c|c|c|c|c|c|}
\hline \multirow[b]{2}{*}{ Local } & \multirow{2}{*}{$\begin{array}{c}\text { Classe } \\
\text { Diamétric } \\
\text { a } \\
\text { Alto Fuste } \\
\text { (40 meses) }\end{array}$} & \multirow{2}{*}{$\begin{array}{l}\% \text { Total } \\
\text { Árvores } \\
\text { (Alto } \\
\text { Fuste) (\%) }\end{array}$} & \multicolumn{6}{|c|}{ Classe Diamétrica Talhadia (40 meses) } \\
\hline & & & Morta (\%) & $0-5 \mathrm{~cm}(\%)$ & $\begin{array}{c}5-10 \mathrm{~cm} \\
(\%)\end{array}$ & $\begin{array}{c}10-15 \mathrm{~cm} \\
(\%)\end{array}$ & $\begin{array}{c}15-20 \mathrm{~cm} \\
(\%)\end{array}$ & $\begin{array}{c}20-25 \mathrm{~cm} \\
(\%)\end{array}$ \\
\hline \multirow{5}{*}{$\begin{array}{c}\text { Belo } \\
\text { Oriente }\end{array}$} & Morta & 1,5 & 100,0 & 0,0 & 0,0 & 0,0 & 0,0 & 0,0 \\
\hline & $5-10 \mathrm{~cm}$ & 1,2 & 17,0 & 0,0 & 25,0 & 41,0 & 17,0 & 0,0 \\
\hline & $10-15 \mathrm{~cm}$ & 94,2 & 6,0 & 2,0 & 12,0 & 53,0 & 26,9 & 0,1 \\
\hline & $15-20 \mathrm{~cm}$ & 3,1 & 3,0 & 3,0 & 10,0 & 31,0 & 53,0 & 0,0 \\
\hline & $20-25 \mathrm{~cm}$ & 0,0 & 0,0 & 0,0 & 0,0 & 0,0 & 0,0 & 0,0 \\
\hline \multicolumn{3}{|c|}{$\%$ de Árvores (Talhadia) } & 7,5 & 1,6 & 11,7 & 51,9 & 27,2 & 0,1 \\
\hline \multirow{5}{*}{$\begin{array}{l}\text { Virginópol } \\
\text { is }\end{array}$} & Morta & 1,8 & 100,0 & 0,0 & 0,0 & 0,0 & 0,0 & 0,0 \\
\hline & $5-10 \mathrm{~cm}$ & 9,9 & 15,0 & 0,0 & 18,0 & 52,0 & 15,0 & 0,0 \\
\hline & $10-15 \mathrm{~cm}$ & 59,7 & 11,0 & 2,0 & 17,6 & 40,0 & 29,0 & 0,4 \\
\hline & $15-20 \mathrm{~cm}$ & 28,6 & 13,0 & 3,0 & 14,0 & 30,0 & 38,0 & 2,0 \\
\hline & $20-25 \mathrm{~cm}$ & 0,0 & 0,0 & 0,0 & 0,0 & 0,0 & 0,0 & 0,0 \\
\hline \multicolumn{3}{|c|}{ \% de Árvores (Talhadia) } & 13,3 & 1,8 & 16,3 & 37,6 & 30,2 & 0,8 \\
\hline
\end{tabular}


A uniformidade de um plantio não é recuperada ao longo do tempo, ocorrendo o seu declínio com o avanço da idade do povoamento (Hakamada et al., 2015). A produção de madeira em nível de povoamento pode diminuir entre 10 e 18\%, devido ao aumento da heterogeneidade do tamanho de árvores em povoamentos de eucalipto (Stape et al., 2010). Em um povoamento clonal, em princípio, todas as plantas possuem a mesma capacidade genética de capturar recursos e convertê-los em estoque de biomassa (Aspinwall et al., 2011). Portanto, a menor produtividade em povoamentos heterogêneos pode ser explicada pela menor eficiência de uso dos recursos (água, luz e nutrientes) dos indivíduos dominados (Ryan et al., 2010). Porém, a uniformidade do volume das árvores em uma plantação pode não ser uma prioridade, se o crescimento das árvores de maior porte for alto o suficiente para compensar o crescimento mais baixo das árvores menores (Canh et al., 2013).

\section{CONCLUSÃO}

Não existe correlação entre as dimensões das variáveis DAP, Ht e volume individual de uma árvore no regime de alto fuste com estas mesmas variáveis da brotação oriunda destas árvores.

A obtenção de um povoamento uniforme em alto fuste não é garantia de um povoamento uniforme na talhadia.

Árvores dominantes no alto fuste podem ser dominadas na talhadia.

\section{REFERÊNCIAS BIBLIOGRÁFICAS}

Andrade, S. D. C., Souza, A. P., Silva, E., Nascimento, A. G., Machado, C. C., Leite, H. G., \& Minetti, L. J. (2000). Analysis of the effects of compaction on regeneration of eucalypt strains. Revista Árvore, 24(3), 261-268.

Aparício, O. S., Ferreira, R. L. C., Silva, J. A. A., Rosa, A. C., \& Aparício, W. C. S. (2010). Controle da matocompetição em plantios de dois clones de Eucalyptus urograndis no Amapá. Ciência Florestal, 20(3), 381-390. http://dx.doi.org/10.5902/198050982053.

Aspinwall, M. J., King, J. S., Mckeand, S. E., \& Bullock, B. P. (2011). Genetic effects on stand-level uniformity and above and belowground dry mass production in juvenile loblolly pine. Forest Ecology and Management, 262(1), 609-619. http://dx.doi.org/10.1016/j.foreco.2011.04.029.

Azevedo, G. B., Sousa, G. T. O., Barreto, P. A. B., \& Conceição Júnior, V. (2011). Estimativas volumétricas em povoamentos de eucalipto sob o regime de alto fuste e talhadia no sudoeste da Bahia. Pesquisa Florestal Brasileira, 31(68), 309-318. http://dx.doi.org/10.4336/2011.pfb.31.68.309.

Cacau, F. V., Reis, G. G., Reis, M. G. F., Leite, H. G., Alves, F. F., \& Souza, F. C. (2008). Decepa de plantas jovens de Eucalipto e manejo de brotações, em um Sistema Agroflorestal. Pesquisa Agropecuária Brasileira, 43(11), 1457-1465. http://dx.doi.org/10.1590/S0100-204X2008001100003.

Canh, T., Binkley, D., \& Luiz, J. (2013). Neighborhood uniformity increases growth of individual Eucalyptus trees. Forest Ecology and Management, 289(1), 90-97.

Christina, M., le Maire, G., Nouvellon, Y., Vezy, R., Bordon, B., Battie-Laclau, P., Gonçalves, J. L. M., DelgadoRojas, J. S., Bouillet, J.-P., \& Laclau, J.-P. (2018). Simulating the effects of different potassium and water supply regimes on soil water content and water table depth over a rotation of a tropical Eucalyptus grandis plantation. Forest Ecology and Management, 418(1), 4-14. http://dx.doi.org/10.1016/j.foreco.2017.12.048.

Dedecek, R. A., \& Gava, J. L. (2005). Influência da compactação do solo na produtividade de rebrota de eucalipto. Revista Árvore, 29(33), 383-390. http://dx.doi.org/10.1590/S0100-67622005000300005.

Faria, G. E., Barros, N. F., Novais, R. F., Lima, J. C., \& Teixeira, J. L. (2002). Produção e estado nutricional de Eucalyptus grandis, em segunda rotação, em resposta à adubação potássica. Revista Árvore, 26(5), 577584. http://dx.doi.org/10.1590/S0100-67622002000500008.

Ferrari, M. P., Ferreira, C. A., \& Silva, H. D. (2004). Condução de plantios de Eucalyptus em sistema de talhadia (28 p.). Colombo: Embrapa Florestas.

Gadelha, F. H. L., Silva, J. A. A., Ferreira, R. L. C., Santos, R. C., \& Tavares, J. A. (2015). Produtividade de clones de eucaliptos em diferentes sistemas de manejo para fins energéticos. Pesquisa Florestal Brasileira, 35(83), 263-270. http://dx.doi.org/10.4336/2015.pfb.35.83.827.

Gonçalves, J. D. M., Alvares, C. A., Behling, M., Alves, J. M., Pizzi, G. T., \& Angeli, A. (2014). Produtividade de plantações de eucalipto manejadas nos sistemas de alto fuste e talhadia, em função de fatores edafoclimáticos. Scientia Forestalis, 42(103), 411-419. 
Guedes, I. C. L., Coelho Júnior, L. M., Oliveira, A. D., Mello, J. M., Rezende, J. L. P., \& Silva, C. P. C. (2011). Economic analysis of replacement regeneration and coppice regeneration in eucalyptus stands under risk conditions. Cerne, 17(3), 393-411. http://dx.doi.org/10.1590/S0104-77602011000300014.

Hakamada, R. E., Stape, J. L., Lemos, C. C. Z., Almeida, A. E. A., \& Silva, L. F. (2015). Uniformity between trees in a full rotation and its relationship with productivity in clonal Eucalyptus. Cerne, 21(3), 465-472. http://dx.doi.org/10.1590/01047760201521031716.

Indústria Brasileira de Árvores - IBÁ. (2017). Relatório IBÁ 2017 (80 p). São Paulo: IBÁ. Recuperado em 11 de dezembro de 2018, de https://iba.org/images/shared/Biblioteca/IBA_RelatorioAnual2017.pdf

Mroz, G. D., Frederick, D. J., \& Jurgensen, M. F. (1985). Site and fertilizer effects on northern hardwood stump sprouting. Canadian Journal of Forest Research, 15(3), 535-543. http://dx.doi.org/10.1139/x85-088.

Otto, M. S. G., Hubbard, R. M., Binkley, D., \& Stape, J. L. (2014). Dominant clonal Eucalyptus grandis x urophylla trees use water more efficiently. Forest Ecology and Management, 328(1), 117-121. http://dx.doi.org/10.1016/j.foreco.2014.05.032.

Reis, G. G., \& Reis, M. G. F. (1997). Fisiologia da brotação de eucalipto com ênfase nas suas relações hídricas. Série Técnica - IPEF, 11(30), 9-22.

Resende, R. T., Soares, A. A. V., Forrester, D. I., Marcatti, G. E., dos Santos, A. R., Takahashi, E. K., e Silva, F. F., Grattapaglia, D., Resende, M. D. V., \& Leite, H. G. (2018). Environmental uniformity, site quality and tree competition interact to determine stand productivity of clonal Eucalyptus. Forest Ecology and Management, 410(1), 76-83. http://dx.doi.org/10.1016/j.foreco.2017.12.038.

Retslaff, F. A., Figueiredo Filho, A., Dias, A. N., Bernett, L. G., \& Figura, M. A. (2015). Curvas de sítio e relações hipsométricas para Eucalyptus grandis na região dos Campos Gerais, Paraná. Cerne, 21(2), 219-225. http://dx.doi.org/10.1590/01047760201521021349.

Rocha, J. H. T., Gonçalves, J. L. M., Gava, J. L., Godinho, T. O., Melo, E. A. S. C., Bazani, J. H., Hubner, A., Arthur Junior, J. C., \& Wichert, M. P. (2016). Forest residue maintenance increased the wood productivity of a Eucalyptus plantation over two short rotations. Forest Ecology and Management, 379(1), 1-10. http://dx.doi.org/10.1016/j.foreco.2016.07.042.

Rode, R., Leite, H. G., Oliveira, M. L. R., Binoti, D. H. B., Ribeiro, C. A. A. S., Souza, A. L., Silva, M. L., \& Cosenza, D. N. (2015). Comparação da regulação florestal de projetos de fomento com áreas próprias de empresas florestais. Pesquisa Florestal Brasileira, 35(81), 11-19. http://dx.doi.org/10.4336/2015.pfb.35.81.760.

Ryan, M. G., Stape, J. L., Binkley, D., Fonseca, S., Loos, R., Takahashi, E. N., Silva, C. R., Silva, S., Hakamada, R., Ferreira, J. M. A., Lima, A. M. N., Gava, J. L., Leite, F. P., Andrade, H. B., Alves, J. M., \& Silva, G. G. C. (2010). Factors controlling Eucalyptus productivity: how resource availability and stand structure alter production and carbono allocation. Forest Ecology and Management, 259(1), 1695-1703. http://dx.doi.org/10.1016/j.foreco.2010.01.013.

Simões, D., Silva, R. B. G., \& Silva, M. R. (2012). Composição do substrato sobre o desenvolvimento, qualidade e custo de produção de mudas de Eucalyptus grandis Hill ex Maiden × Eucalyptus urophylla S. T. Blake. Ciência Florestal, 22(1), 91-100. http://dx.doi.org/10.5902/198050985082.

Stape, J. L., Binkley, D., Ryan, M. G., Fonseca, S., Loos, R. A., Takahashi, E. N., Silva, C. R., Silva, S. R., Hakamada, R. E., Ferreira, J. M. A., Lima, A. M. N., Gava, J. L., Leite, F. P., Andrade, H. B., Alves, J. M., Silva, G. G. C., \& Azevedo, M. R. (2010). The Brazil eucalyptus potential productivity project: influence of water, nutrients and stand uniformity on wood production. Forest Ecology and Management, 259(1), 1684-1694. http://dx.doi.org/10.1016/j.foreco.2010.01.012.

Statsoft Inc. (2014). Statistica (data analysis software system), version 12. Recuperado em 22 de abril de 2018, de www.statsoft.com

Teixeira, P. C., Novais, R. F., Barros, N. F., Neves, J. C. L., \& Teixeira, J. L. (2002). Eucalyptus urophylla root growth, stem sprouting and nutrient supply from the roots and soil. Forest Ecology and Management, 160(3), 263-271.

Walters, J. R., Bell, T. L., \& Read, S. (2005). Intraspecific variation in carbohydrate reserves and sprouting ability in Eucalyptus obliqua seedlings. Australian Journal of Botany, 53(3), 195-203. http://dx.doi.org/10.1071/BT04016.

Contribuição dos Autores: GMPF: Escrita - Primeira Redação, Escrita - Revisão e Edição, Metodologia, Curadoria de Dados, Conceituação; LAGJ: Primeira Redação, Escrita - Revisão e Edição, Metodologia; BLSS: Primeira Redação, Escrita - Revisão e Edição, Metodologia; HNP: Escrita - Revisão e Edição, Metodologia; FPL: Metodologia; PHV: Escrita - Revisão e Edição, Curadoria de Dados; SJSSR: Escrita - Revisão e Edição, Curadoria de Dados; HGL: Escrita - Revisão e Edição. 